\title{
Sacubitril/valsartan in everyday clinical practice: an observational study based on the experience of a heart failure clinic
}

\author{
Joana Cabral ${ }^{1,2}$, Henrique Vasconcelos ${ }^{1,2}$, Paulo Maia-Araújo ${ }^{2,3,4}$, Emília Moreira ${ }^{2,5}$, Manuel Campelo ${ }^{2,3,4}$, \\ Sandra Amorim ${ }^{2,3,4}$, Alexandra Sousa ${ }^{2,4,6}$, Brenda Moura ${ }^{2,4,7}$, Roberto Pinto ${ }^{3,8}$, Camila Dias ${ }^{4,5}$, \\ José Silva-Cardoso ${ }^{2,3,4} \wedge$
}

${ }^{1}$ Faculty of Medicine, University of Porto, Porto, Portugal; ${ }^{2}$ CINTESIS-Center for Health Technology and Services Research, Porto, Portugal; ${ }^{3}$ Department of Cardiology, Centro Hospitalar Universitário de São João, Porto, Portugal; ${ }^{4}$ Department of Medicine, Faculty of Medicine, University of Porto, Porto, Portugal; ${ }^{5}$ Department of Community Medicine, Information and Health Decision Sciences, Faculty of Medicine, University of Porto, Porto, Portugal; ${ }^{6}$ Department of Cardiology, Hospital Santa Maria Maior, Barcelos, Portugal; ${ }^{7}$ Department of Cardiology, Hospital das Forças Armadas, Polo do Porto, Porto, Portugal; ${ }^{8}$ Department of Biomedicine, Faculty of Medicine, University of Porto, Porto, Portugal

Contributions: (I) Conception and design: J Silva-Cardoso, E Moreira, J Cabral, H Vasconcelos, P Maia-Araújo; (II) Administrative support: E Moreira; (III) Provision of study materials or patients: J Silva-Cardoso, R Pinto, A Sousa, B Moura, P Maia-Araújo, M Campelo, S Amorim; (IV) Collection and assembly of data: J Cabral, H Vasconcelos, E Moreira; (V) Data analysis and interpretation: J Silva-Cardoso, E Moreira, J Cabral, H Vasconcelos, C Dias; (VI) Manuscript writing: All authors; (VII) Final approval of manuscript: All authors.

Correspondence to: José Silva-Cardoso. CINTESIS-Center for Health Technology and Services Research, Rua Dr. Plácido da Costa, Porto, Portugal. Email: silvacardoso30@gmail.com.

Background: Heart failure (HF) is a growing public health problem. Sacubitril/valsartan is now recommended to be used in persistently symptomatic patients with left ventricular ejection fraction (LVEF) $<40 \%$, replacing angiotensin-converting enzyme inhibitors (ACEis)/angiotensin receptor blockers (ARBs). In the present study, we aimed to characterise the challenges of sacubitril/valsartan use in everyday clinical practice.

Methods: We assessed the medical records of patients with HF and reduced ejection fraction eligible for sacubitril/valsartan attending a HF clinic at a Portuguese University Hospital during 2018 ( $\mathrm{n}=152$ ). The number of eligible patients receiving the drug and the reasons for not prescribing sacubitril/valsartan were evaluated. Additionally, we assessed the tolerability of maximal doses of sacubitril/valsartan. New York Heart Association functional class (NYHA class) and LVEF before and after up-titration to maximal tolerated sacubitril/valsartan dose were compared. Median follow-up was 41 months.

Results: Of the 152 included patients, 75 (49\%) were prescribed the drug. The two main reasons for non-prescription were patient financial barriers (31\%) and hypotension (27\%). Only 33\% of patients on sacubitril/valsartan did reach maximal dose. Hypotension was the main limiting factor for dose optimisation. Duration of sacubitril/valsartan treatment showed a positive association with LVEF improvement during follow-up (6.6\% absolute LVEF increase/year). NYHA functional class improved significantly from baseline through the end of follow-up.

Conclusions: In every-day clinical practice, although sacubitril/valsartan was associated with a marked improvement in NYHA class and in LVEF, important financial and clinical barriers to the implementation of this therapy were identified.

Keywords: Sacubitril/valsartan; heart failure (HF); neurohormonal modulation; real-life practice; financial barriers

Submitted May 12, 2021. Accepted for publication Sep 29, 2021.

doi: $10.21037 / \mathrm{cdt}-21-312$

View this article at: https://dx.doi.org/10.21037/cdt-21-312

^ ORCID: 0000-0002-9774-9864. 


\section{Introduction}

Heart failure (HF) is a growing public health problem with 26 million people worldwide being affected (1). Its prevalence is estimated to increase by $25 \%$ during the next decade (2). HF represents a heavy economic burden mainly due to hospitalizations (1). The latter are mainly associated with increased morbidity and mortality (3). In fact, it has been reported that $\mathrm{HF}$ mortality is higher than that of most common cancers $(4,5)$.

Triple neurohormonal blockade, with the combination of an angiotensin-converting enzyme inhibitor (ACEi) or an angiotensin receptor blocker (ARB), a $\beta$-blocker (BB) and a mineralocorticoid receptor antagonist (MRA) was the standard therapy for $\mathrm{HF}$ with reduced ejection fraction (HFrEF) until the PARADIGM-HF study was published in 2014 (6). In PARADIGM-HF, sacubitril/valsartan, an angiotensin-receptor/neprilysin inhibitor (ARNI), reduced cardiovascular mortality and $\mathrm{HF}$ hospitalizations compared to enalapril (6). The findings of this study established neurohormonal modulation as the new paradigm in $\mathrm{HFrEF}$ therapy (7). In fact, sacubitril/valsartan not only blocks the renin-angiotensin-aldosterone system (a regulatory system) via valsartan but, additionally, enhances the natriuretic peptides system (a counter-regulatory system) via sacubitril (8).

Current HFrEF guidelines recommend that, in persistently symptomatic patients, ACEis/ARBs should be replaced by sacubitril/valsartan $(7,9)$.

Patients included in randomized clinical trials such as PARADIGM-HF are frequently selected according to strict inclusion and exclusion criteria. Thus, they do not fully represent the HF-population found in everyday clinical practice (10-12). This highlights the relevance of studies conducted in real-life settings, to identify possible barriers to the implementation of therapeutic strategies developed in more controlled conditions.

The present study evaluated the use of sacubitril/ valsartan in real-life clinical practice conditions. It was conducted in a University Hospital outpatient HF-Clinic. We analysed sacubitril/valsartan rates of prescription, dosage, prescription-limiting factors and down-titration or discontinuation factors. We aimed to identify barriers to the full implementation of sacubitril/valsartan in daily clinical practice.

We present the following article in accordance with the STROBE reporting checklist (available at https://dx.doi. org/10.21037/cdt-21-312).

\section{Methods}

\section{Study design}

This is a retrospective observational study conducted at an outpatient HF-Clinic of a Portuguese University Hospital Centre.

\section{Setting}

All consecutive patients with HFrEF attending the Heart Failure Clinic of the Cardiology Department of S. João University Hospital (CHUSJ), Porto, Portugal, during 2018 were screened for inclusion. Each patient's 2018 first appointment was defined as the patient's index visit in terms of the present study. After inclusion into the study, each patient's medical records occurring from January 1st, 2016 up to December 31st, 2019 were reviewed in order to encompass each patient's total time under sacubitril/ valsartan. All procedures were conducted in accordance with the tenets of the Declaration of Helsinki (as revised in 2013). The protocol was approved by the S. João University Hospital Health Ethics Committee (No. 428-19). Since this was a study based on secondary data collection, the need for a written informed consent was waived by the Ethics Committee.

\section{Participants}

Patients were considered candidates for sacubitril/ valsartan therapy if they were 18 years or older, with $\mathrm{HF}$ diagnosis according to the 2016 European Society of Cardiology (ESC) Heart Failure Guidelines (7), with left ventricular ejection fraction (LVEF) $<40 \%$ evaluated by 2D-echocardiography, and symptomatic (NYHA class II-IV), despite optimal therapy with ACEi or ARB in addition to a beta-blocker and an MRA, at any appointment in the follow-up period, as per current guidelines $(7,9)$. Patients in NYHA I at the date of sacubitril/valsartan prescription were excluded from this study. No other exclusion criteria were applied.

\section{Variables}

Quantitative and qualitative data were collected from each patient's electronic health records, including age, sex, HF aetiology, NYHA functional class, HF signs and symptoms, 
echocardiographic data, blood and urine laboratory data and medication history. The presence of comorbidities, namely diabetes mellitus, dyslipidaemia, hypertension, and renal failure was registered when explicitly stated in clinical records. The primary outcome was the proportion of sacubitril/valsartan candidates who were prescribed with this drug. We have also assessed the reasons preventing patients from undergoing optimal therapy. Additionally, we explored the evolution in LVEF, left ventricular enddiastolic volume and mass, NYHA class, congestion signs and estimated glomerular filtration rate (eGFR) in patients treated with sacubitril/valsartan.

\section{Data sources management}

Only laboratory data originated within a 3-month period window regarding each appointment and echocardiographic data originated within a 6 -month window regarding the appointment were considered. Obesity was defined by a body mass index $(\mathrm{BMI})>30 \mathrm{~kg} / \mathrm{m}^{2}$.

Renal failure was considered clinically relevant only in patients presenting chronic kidney disease stage $\geq 3$ (eGFR $<60 \mathrm{~mL} / \mathrm{min} / 1.73 \mathrm{~m}^{2}$ ), evaluated by the Chronic Kidney Disease Epidemiology Collaboration (CKDEPI) formula. Medication records were reviewed. For each neurohormonal blocker, the dose was converted into the equivalent of a reference drug: enalapril for ACEis, candesartan for ARBs, bisoprolol for BBs, and spironolactone for MRAs. The target dose of sacubitril/ valsartan was the one referred on the 2016 ESC HF guidelines (7) of 97/103 mg bid. Reasons for not prescribing sacubitril/valsartan, or for sacubitril/valsartan dose uptitration failure, dose-reduction, or drug withdrawal were collected from clinical files or marked as unknown when no information was available.

\section{Bias}

This study was only performed in one Heart Failure Clinic from the North of Portugal. This may constitute a source of bias.

\section{Study size}

The study includes all the patients that attended the Heart Failure Clinic of the Cardiology Department of S. João University Hospital (CHUSJ), Porto, Portugal, during 2018.

\section{Statistical analysis}

Statistical analyses and data management were performed with MS Excel and R Studio using R software, version 3.6.2 (R Foundation for Statistical Computing). Continuous variables were expressed as mean and standard deviation when normally distributed, and as median and interquartile range (IQR) otherwise. Categorical variables were summarized as counts and percentage of total sample for each class. Missing data cases were excluded from the number of cases in each calculation. A linear regression model was used to assess the change in LVEF over time after initiation of sacubitril/valsartan. The Wilcoxon pairedsamples sign rank test was used to assess differences in the distribution of NYHA class and in eGFR in patients before and after treatment with sacubitril/valsartan. McNemar test was used to assess the difference in the presence of congestion signs before and after treatment with sacubitril/ valsartan. Statistical significance was set at $\mathrm{P}<0.05$, twotailed.

\section{Results}

\section{Patients' characteristics at the 2018 index visit}

A total of 537 patients with HF were seen at the outpatient HF-Clinic during 2018. Overall, 152 patients met our inclusion criteria. The median LVEF was $28 \%$ (IQR, $22-33 \%)$. Patients' characteristics at index visit are listed in Table 1. Mean age was $62.2 \pm 9.9$ years and $105(69 \%)$ were men.

In $64(42 \%)$ patients, HF aetiology was ischaemic heart disease. Regarding cardiovascular risk factors, 107 $(70 \%)$ of patients had dyslipidaemia, $80(53 \%)$ a smoking history, 73 (49\%) hypertension, 58 (38\%) diabetes mellitus and $53(37 \%)$ were obese. In 44 (34\%) patients eGFR was $<60 \mathrm{~mL} / \mathrm{min} / 1.73 \mathrm{~m}^{2}$.

Patients were followed up for a median of 40.6 (IQR, 29.835.8) months. Each patient attended a median of 3.2 (IQR, 2.6-4.4) appointments per year. The median time of treatment with sacubitril/valsartan was 10.4 (IQR, 6.8-15.7) months.

\section{Eligible patients prescribed with sacubitril/valsartan and reasons preventing optimal therapy}

Only 75 (49\% of all candidates) were on sacubitril/valsartan at the index visit (Figure 1).

The main reasons for non-prescription were financial barriers (31\%), hypotension (27\%) and renal failure (14\%). 
Table 1 Patient characteristics at the 2018 index visit

\begin{tabular}{|c|c|}
\hline Variables & Value \\
\hline Age (years), mean $\pm S D$ & $62.2 \pm 9.9$ \\
\hline Male sex, n (\%) & $105 / 152(69.1)$ \\
\hline BMI $\left(\mathrm{kg} / \mathrm{m}^{2}\right)$, mean $\pm \mathrm{SD}$ & $28.5 \pm 4.9$ \\
\hline Ischaemic aetiology, n (\%) & $64 / 152(42.1)$ \\
\hline \multicolumn{2}{|l|}{ Risk factors, n (\%) } \\
\hline Dyslipidaemia & 107/152 (70.4) \\
\hline Hypertension & $73 / 150(48.7)$ \\
\hline Obesity & $53 / 142(37.3)$ \\
\hline Ex-smoker & $56 / 152(36.8)$ \\
\hline Current smoker & 24/152 (15.8) \\
\hline Diabetes mellitus & $58 / 151(38.4)$ \\
\hline \multicolumn{2}{|l|}{ NYHA class*, n (\%) } \\
\hline I & $9 / 151(6.0)$ \\
\hline II & $107 / 151(70.9)$ \\
\hline III & $31 / 151(20.5)$ \\
\hline IV & 4/151 (2.6) \\
\hline $\mathrm{SBP}(\mathrm{mmHg})$, mean $\pm \mathrm{SD}$ & $116.1 \pm 17.3$ \\
\hline $\mathrm{DBP}(\mathrm{mmHg})$, mean $\pm \mathrm{SD}$ & $67.8 \pm 10.4$ \\
\hline $\mathrm{HR}(\mathrm{bpm})$, mean $\pm \mathrm{SD}$ & $67.8 \pm 10.8$ \\
\hline BNP (pg/mL), median (IQR) & $156.0(91.5-290.0)$ \\
\hline LVEF (\%), median (IQR) & $28.0(22.0-33.0)$ \\
\hline Creatinine (mg/dL), median (IQR) & $0.9(0.8-1.1)$ \\
\hline \multicolumn{2}{|l|}{ CKD stage, n (\%) } \\
\hline 1 & 33/131 (25.2) \\
\hline 2 & $50 / 131(38.2)$ \\
\hline 3 & $37 / 131$ (28.2) \\
\hline 4 & $7 / 131(5.3)$ \\
\hline 5 & $0 / 31(0.0)$ \\
\hline Potassium (mmol/L), mean \pm SD & $4.5 \pm 0.6$ \\
\hline \multicolumn{2}{|l|}{ Medication, n (\%) } \\
\hline ACEis & $116 / 152(76.3)$ \\
\hline ARBs & $24 / 152(15.8)$ \\
\hline BBs & $144 / 152(94.7)$ \\
\hline MRAs & 134/152 (88.2) \\
\hline $\mathrm{ARNi}$ & 7/152 (4.6) \\
\hline
\end{tabular}

*, the data for NYHA class reflect the status of patients at the time of index visit. Patients were required to have displayed at least NYHA class II symptoms at any point of the follow-up period. BMI, body mass index; NYHA, New York Heart Association; SBP, systolic blood pressure; DBP, diastolic blood pressure; HR, heart rate; BNP, B-type natriuretic peptide; LVEF, left ventricular ejection fraction; CKD, chronic kidney disease; ACEi, angiotensin-converting enzyme inhibitor; $A R B$, angiotensin II receptor blocker; BB, $\beta$-blocker; MRA, mineralocorticoid receptor antagonist; $\mathrm{ARNi}$, angiotensin receptor-neprilysin inhibitor; SD, standard deviation; IQR, interquartile range.
In 17 (22\%) patients no cause was listed for sacubitril/ valsartan non-prescription. Results are summarised in Table 2.

Twenty-one of the 75 patients on sacubitril/valsartan were still on drug up-titration at the end of follow-up and were withdrawn from further analysis as they had still not reached their maximal tolerated dose (Figure 2).

Of the remaining patients, $18(33 \%)$ tolerated the sacubitril/valsartan target dose of $97 / 103 \mathrm{mg}$ bid, and $32(59 \%)$ patients were under a suboptimal sacubitril/ valsartan dose. In 4 (7\%) patients sacubitril/valsartan was discontinued.

Reasons for sacubitril/valsartan dose-downgrading, withdrawal or inability to reach target dose $(97 / 103 \mathrm{mg}$ bid) were hypotension (53\%), hyperkalemia (14\%) and renal failure (11\%) (Table 3). Other reasons $(8 \%)$ included diarrhoea and financial barriers. In $14 \%$ of patients no reason for suboptimal dose was identified.

\section{LVEF, NYHA class, congestion signs and renal function changes in patients under sacubitril/valsartan}

We observed a positive association between the duration of sacubitril/valsartan treatment and the magnitude of LVEF improvement and of left ventricle (LV) end-diastolic volume reduction during follow-up. A linear regression model evidenced an absolute 6.6\% LVEF increase per year of sacubitril/valsartan treatment $(\mathrm{P}=0.012)$ (see Appendix 1, Figure S1) and an absolute $58 \mathrm{~mL}$ decrease in left ventricular end-diastolic volume $(\mathrm{P}=0.025)$. No other statistically significant echocardiographic changes were observed during follow-up, an observation that must be taken with caution taking into account the limited number of patients.

In patients under sacubitril/valsartan for a minimum of 6 months a statistically significant improvement in NYHA class was observed $(\mathrm{P}<0.001)$ (Figure 3).

No significant difference was found between the presence of signs of congestion at baseline visit and at the end of follow-up $(\mathrm{P}=0.109)$.

eGFR median values showed a slight decrease between the beginning of treatment with sacubitril/valsartan and the end of follow-up [median $=79(\mathrm{IQR}, 65-93) \mathrm{mL} / \mathrm{min} / 1.73 \mathrm{~m}^{2}$ vs. median $\left.=78(\mathrm{IQR}, 53-92) \mathrm{mL} / \mathrm{min} / 1.73 \mathrm{~m}^{2} ; \mathrm{P}=0.017\right]$.

\section{Discussion}

In our retrospective study, we included all candidates for sacubitril/valsartan attending an outpatient HF-Clinic 


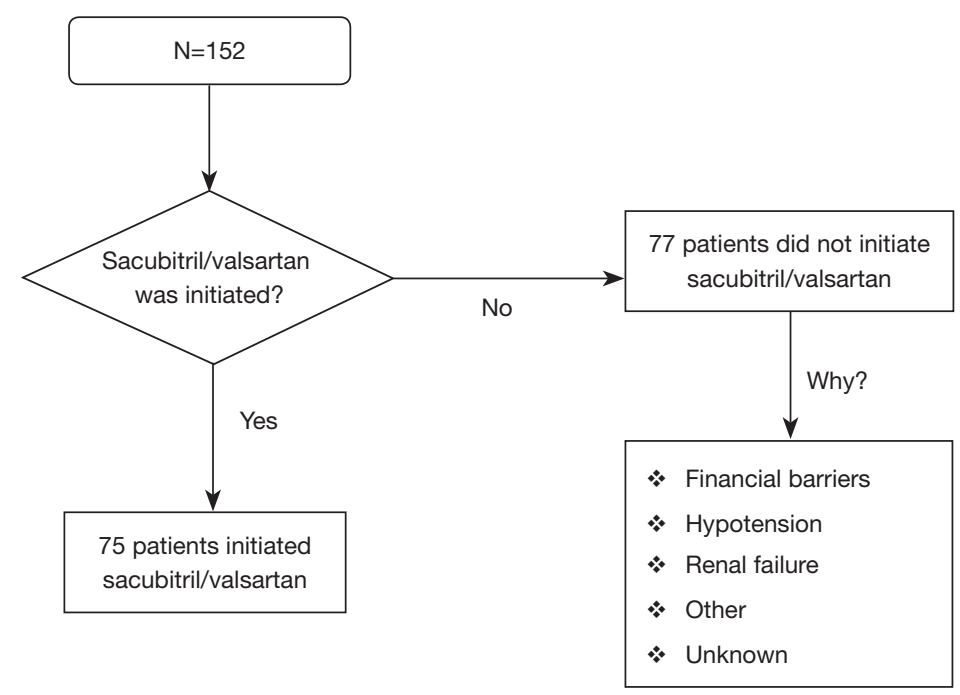

Figure 1 Disposition of patients during the study.

Table 2 Reasons for sacubitril/valsartan non-prescription among candidates who were not on the drug

\begin{tabular}{lc}
\hline Reason for non-prescription & Patients $(\mathrm{n}=77), \mathrm{n}(\%)$ \\
\hline Financial barriers & $24(31.2)$ \\
Hypotension & $21(27.3)$ \\
Renal failure stage $\geq 3^{*}$ & $11(14.3)$ \\
eGFR $<30 \mathrm{~mL} / \mathrm{min} / 1.73 \mathrm{~m}^{2}$ & $6(7.8)$ \\
Other & $4(5.2)$ \\
Unknown/not listed & $17(22.1)$ \\
\hline
\end{tabular}

*, eGFR $<60 \mathrm{~mL} / \mathrm{min} / 1.73 \mathrm{~m}^{2}$; \#, age and adverse effects, including cough and diarrhoea. eGFR, estimated glomerular filtration rate.

of a University Hospital Centre during 2018 ( $\mathrm{n}=152)$. After inclusion in the study, all patients' medical records from January 1st, 2016 up to December 31st, 2019 were reviewed.

During that 4 -year period, only $49 \%$ of the candidates initiated the drug. The main reasons for sacubitril/valsartan non-prescription were financial barriers, hypotension and renal failure. In $22 \%$ of patients no cause was listed for sacubitril/valsartan non-prescription.

Only $33 \%$ of the patients on sacubitril/valsartan tolerated the $97 / 103 \mathrm{mg}$ bid target dose. The main reasons for sacubitril/valsartan dose-downgrading, drug withdrawal or inability to reach $97 / 103 \mathrm{mg}$ bid were hypotension, hyperkalemia and renal failure.
An absolute 6.6\% LVEF increase per year of sacubitril/ valsartan treatment was observed, as well as a significant improvement in NYHA class in those under the drug for a minimum of 6 months.

\section{PARADIGM-HF and PROVE-HF studies}

In the landmark controlled, double-blinded randomized PARADIGM-HF study $(\mathrm{n}=8,399 ; 1,043$ participating centres) sacubitril/valsartan proved to reduce cardiovascular mortality, total mortality, HF hospitalizations (6) and clinical deterioration (13) compared to enalapril. In addition to a positive impact in NYHA functional class (13), more recent evidence derived from the prospective, 12-month follow-up, single-group, open-label PROVE-HF study ( $\mathrm{n}=794 ; 78$ participating centres) showed a positive impact of sacubitril/valsartan on LVEF and LV remodelling in HFrEF patients (14).

\section{Differences and similarities amongst the 3 studies}

Comparison of the above 2 studies and our study show some differences in setting, design, population size and objectives (Table 4), albeit also some similarities. Patients' age and sex, and baseline LVEF, natriuretic peptides and creatinine were similar. HF aetiology in PARADIGM-HF and in PROVEHF was more frequently ischemic heart disease, and patients were more frequently hypertensive than in our study. Patients in PROVE-HF had a higher BMI and were more frequently 


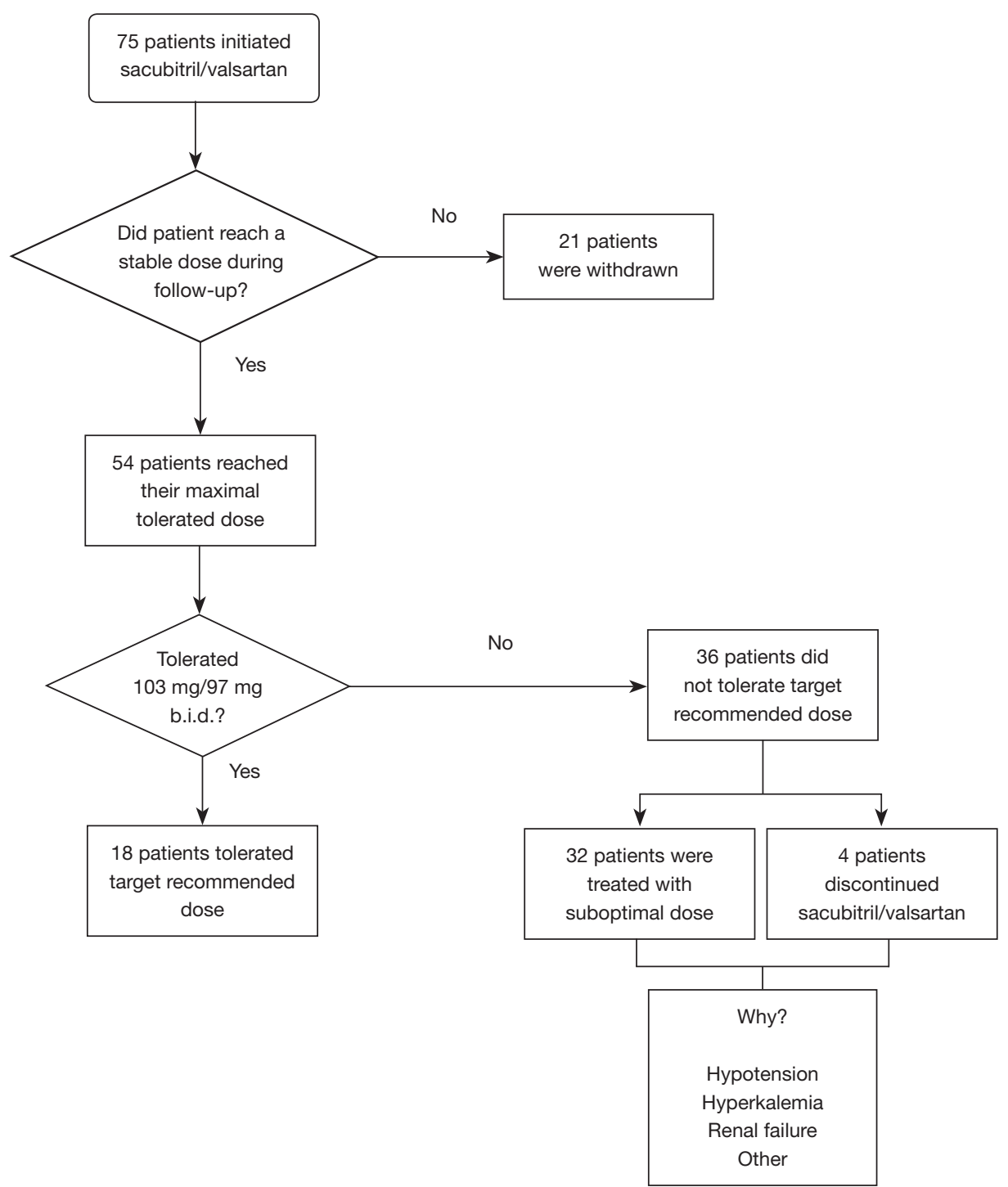

Figure 2 Disposition of patients according to tolerability of target recommended sacubitril/valsartan doses.

Table 3 Reasons for sacubitril/valsartan under-titration among patients not tolerating target recommended dose

\begin{tabular}{lc}
\hline Reason for under-titration & Patients $(\mathrm{n}=36)^{\star}, \mathrm{n}(\%)$ \\
\hline Hypotension & $19(52.8)$ \\
Hyperkalemia & $5(13.9)$ \\
Unknown & $5(13.9)$ \\
Renal Failure & $4(11.1)$ \\
Other & $3(8.3)$ \\
\hline
\end{tabular}

*, patients still under sacubitril/valsartan up-titration at the end of follow-up were withdrawn from this analysis. diabetic than in PARADIGM-HF and in our study.

\section{Patients not prescribed with sacubitril/valsartan}

In all 3 studies it is noticeable that a sizable proportion of candidates for sacubitril/valsartan were not prescribed with this therapy and this occurred more frequently in our case. In our study, $51 \%$ of the candidates for sacubitril/valsartan did not initiate the drug. This was attributed to financial barriers, hypotension or renal failure. In almost one quarter of patients who did not initiate sacubitril/valsartan, no cause 


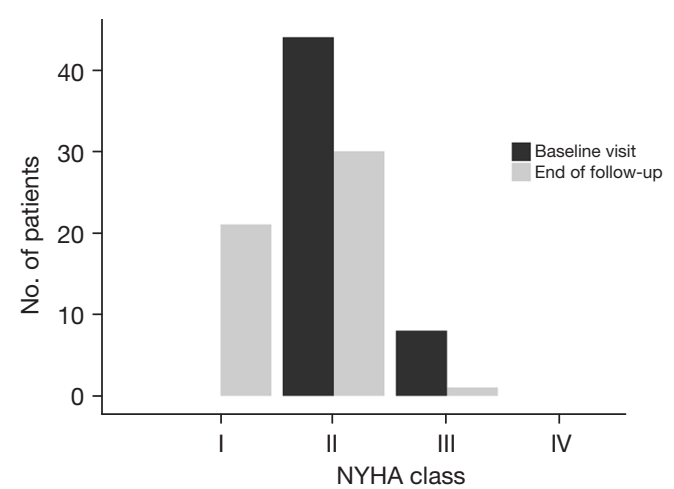

Figure 3 Changes in NYHA functional class in patients who were under therapy with sacubitril/valsartan for at least 6 months. NYHA, New York Heart Association.

was listed for non-prescription.

Current ESC guidelines contraindicate sacubitril/valsartan only in patients with stage 5 renal failure (7). In our case, chronic kidney disease was a frequent reason for sacubitril/ valsartan non-prescription, even though only half of the patients had an eGFR under $30 \mathrm{~mL} / \mathrm{min} / 1.73 \mathrm{~m}^{2}$. This inconsistency with what is proposed by the ESC guidelines may represent the physicians' reluctance to tap into uncharted areas of clinical response to a relatively new drug.

In PARADIGM-HF, during the run-in period, $12 \%$ of the patients stopped sacubitril/valsartan because of an adverse event, most frequently cough, hyperkalemia, hypotension or renal dysfunction (6). After randomization, except for discontinuations owing to death, sacubitril/ valsartan was discontinued in another $18 \%$ due to several reasons, which in $11 \%$ of all patients $(60 \%$ of all that discontinued sacubitril/valsartan), was an adverse event (6). In PROVE-HF $18 \%$ of patients stopped sacubitril/valsartan prematurely (14).

It is clear that sacubitril/valsartan may need to be discontinued for a number of clinical reasons as hyperkalemia, hypotension, or renal dysfunction and these side-effects must be cautiously monitored. However, financial barriers for the implementation of this life-saving therapy, as occurred in our case, are unacceptable from an ethical standpoint, especially given that sacubitril/valsartan has proved to be cost-effective $v$ s. enalapril in patients with HFrEF (15-20). The magnitude of this problem should promote a wide discussion over the co-payment share of sacubitril/valsartan.

The absence of an explicit reason not to initiate sacubitril/valsartan in $22 \%$ of patients not on the drug, observed in our study, may reflect therapeutic inertia and requires special attention.

\section{Treatment doses}

In PARADIGM-HF, sacubitril/valsartan mean total daily dose at last assessment was $375 \pm 71 \mathrm{mg}$. In PROVE-HF, by the end of the study, the maximum sacubitril/valsartan dose was $97 / 103 \mathrm{mg}$ bid in $65 \%$ patients, $49 / 51 \mathrm{mg}$ bid in $21 \%$, and $24 / 26 \mathrm{mg}$ bid in $14 \%$. Reasons for not reaching target doses in PROVE-HF included symptomatic hypotension in $5 \%$, kidney dysfunction in $2 \%$, and hyperkalemia in $1 \%$ of patients. The much lower rate of target doses of sacubitril/valsartan amongst our patients, as compared to PARADIGM-HF and PROVE-HF, may reflect the higher prevalence of hypertension in PARADIGM-HF and in PROVE-HF and the higher BMI of PROVE-HF patients as compared to our population. Additionally, the aggressive titration protocol used in PARADIGM-HF with dose upscaling every 2 weeks under blood pressure, creatinine and serum potassium control may be difficult to replicate in the everyday clinical conditions of a real-world HF-clinic. Nevertheless, and again, an element of clinical inertia and/ or reluctance due to adverse events may be present and must be addressed.

\section{Other prescription constraints}

Several patients' records lacked a reason for sub- or nonprescription of sacubitril/valsartan. We draw on some of the characteristics of real-life clinical practice in a tertiary hospital to provide a possible explanation for this.

Firstly, the pressure associated with an overloaded appointment schedule, allowing for very little time in each visit, partially decreases the quality of provided care. Secondly, the logistic mismatch between the time of the visits and the availability of exams' results often prevents the physician from having a safe ground to prescribe at an ideal pace. Thirdly, the lack of a clearly defined protocol for the management of patients in this clinic may contribute to insecurity in further advancing the treatment of an apparently stabilised patient. Lastly, the belief that a patient who consistently shows no deterioration of their status is stabilised may prove dangerous, as some of them may rapidly evolve to develop a fatal arrhythmia from a supposedly innocuous NYHA II background. 
Table 4 Patient characteristics at index visit in present study, PARADIGM-HF and PROVE-HF

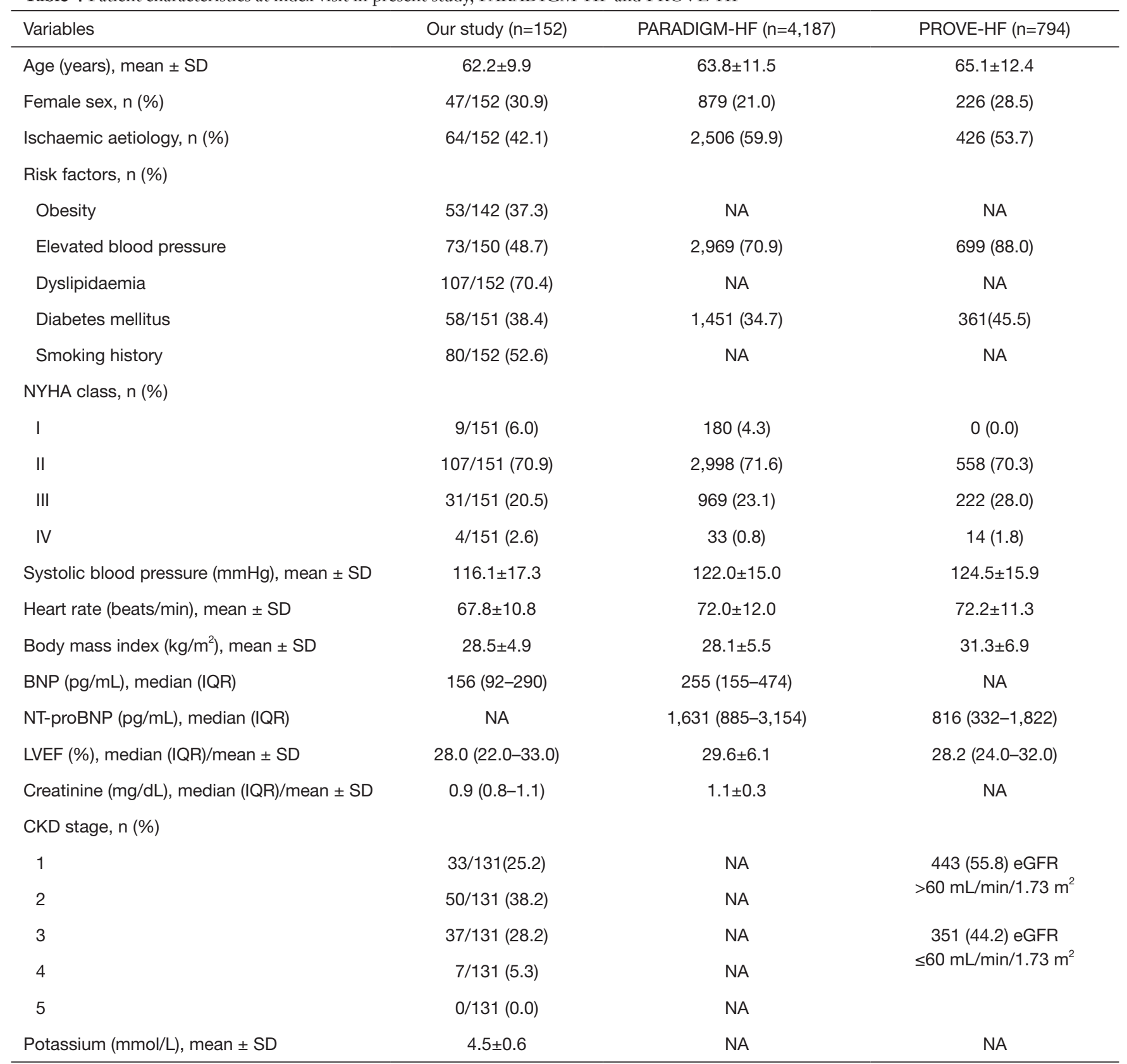

SD, standard deviation; NA, not available; NYHA, New York Heart Association; BNP, B-type natriuretic peptide; IQR, interquartile range; NT-proBNP, N-terminal pro-B-type natriuretic peptide; LVEF, left ventricular ejection fraction; CKD, chronic kidney disease; eGFR, estimated glomerular filtration rate.

\section{Impact in patients under sacubitril/valsartan}

It is frequently argued that randomized controlled clinical trials' populations are often over-selected and may not represent the real-life populations for which the tested drug is clinically indicated (10-12). Our study analysed a real-life clinical setting with all the constraints found in everyday clinical practice. Despite all the above, it is worth noticing that a positive impact on soft endpoints such as NYHA functional class and LVEF was replicated in our unselected (all-comers) population. This is in line with data 
derived from PARADIGM-HF (13) and the observed data in PROVE-HF (14). The impact of sacubitril/valsartan on LVEF in HFrEF may be explained by its potential to blunt adverse cardiac remodeling in $\mathrm{HFrEF}$, as demonstrated in previous studies (21).

Although there is evidence supporting an improvement in clinical signs of congestion with sacubitril/valsartan (22), no significant difference was observed in our study. This may be explained by the fact that most patients in our study did not show signs of congestion in the analysed outpatient visits.

Finally, in our study, patients treated with sacubitril/ valsartan showed a decline in eGFR. This is consistent with previous clinical trials with sacubitril/valsartan $(23,24)$ and is an expected result, since a worsening renal function is frequent in HF patients (25).

\section{Limitations}

This study has several limitations. Firstly, its observational, single-group design hampers group comparisons or effect assessment. Secondly, data was retrospectively collected from records whose main purpose was not clinical research, and that posed problems regarding missing and incomplete variables. Particularly, parameters such as $\mathrm{N}$-terminal pro-B-type natriuretic peptide (NT-proBNP) levels and quality of life indicators were not available as they were not assessed by routine at our clinic. Thirdly, accounting for cardiac resynchronization therapy defibrillator (CRT-D) and implantable cardioverter defibrillator (CDI) devices and controlling for the long-term effect of previous ACEi/ARB therapy and their potential influence in estimating gains with sacubitril/valsartan therapy in real life clinical settings has not been considered. Similarly, it should be noted that simultaneous therapy with SGLT2 inhibitors was not assessed, although they have recently been demonstrated to improve systolic and diastolic function in $\operatorname{HF}(26,27)$. Fourthly, having conducted this study in a single centre raises difficulties when generalising the findings to a broader population, such as the entire Portuguese HFrEF population.

\section{Conclusions}

In everyday clinical conditions, although sacubitril/valsartan was associated with a marked improvement in NYHA functional class and in LVEF, important financial and clinical barriers to the implementation of this therapy were identified.

\section{Acknowledgments}

The authors would like to thank Constança Coelho PhD from $\mathrm{x} 2$-Science Solutions for the support in medical writing, review, and submission of this manuscript.

Funding: This work was supported by Novartis, via funding for third-party medical writing, review, and submission of this manuscript.

\section{Footnote}

Reporting Checklist: The authors have completed the STROBE reporting checklist. Available at https://dx.doi. org/10.21037/cdt-21-312

Data Sharing Statement: Available at https://dx.doi. org/10.21037/cdt-21-312

Conflicts of Interest: All authors have completed the ICMJE uniform disclosure form (available at https://dx.doi. org/10.21037/cdt-21-312). All authors report that this work was supported by Novartis, via funding for third-party medical writing, review, and submission of this manuscript. AS has received speaker fees or advisory board participation fees from MSD, Merck Serono, Novartis, Servier, Bial and Pfizer outside of this work. BM has received speaker fees from AstraZeneca, Boehringer Ingelheim, Lilly, Servier, Novartis and Merck Serono outside of this work. JSC has received speaker and consultant fees, or advisory board participation fees from Abbott, AstraZeneca Pharmaceuticals, Bial, Boehringer Ingelheim, Menarini, Merck Serono, Merck Sharp \& Dohme, Novartis, Orion, Pfizer, Sanofi, Servier, and Vifor Pharma outside of this work. The authors have no other conflicts of interest to declare.

Ethical Statement: The authors are accountable for all aspects of the work in ensuring that questions related to the accuracy or integrity of any part of the work are appropriately investigated and resolved. All procedures were conducted in accordance with the tenets of the Declaration of Helsinki (as revised in 2013). The protocol was approved by the S. João University Hospital Health Ethics Committee. The protocol was approved by the S. João University Hospital Health Ethics Committee (No. 428-19). Since this was a study based on secondary data collection, the need for a written informed consent was waived by the Ethics Committee. 
Open Access Statement: This is an Open Access article distributed in accordance with the Creative Commons Attribution-NonCommercial-NoDerivs 4.0 International License (CC BY-NC-ND 4.0), which permits the noncommercial replication and distribution of the article with the strict proviso that no changes or edits are made and the original work is properly cited (including links to both the formal publication through the relevant DOI and the license). See: https://creativecommons.org/licenses/by-nc-nd/4.0/.

\section{References}

1. Ponikowski P, Anker SD, AlHabib KF, et al. Heart failure: preventing disease and death worldwide. ESC Heart Fail 2014;1:4-25.

2. Mazurek JA, Jessup M. Understanding Heart Failure. Card Electrophysiol Clin 2015;7:557-75.

3. Jaagosild P, Dawson NV, Thomas C, et al. Outcomes of acute exacerbation of severe congestive heart failure: quality of life, resource use, and survival. SUPPORT Investigators. The Study to Understand Prognosis and Preferences for Outcomes and Risks of Treatments. Arch Intern Med 1998;158:1081-9.

4. Stewart S, MacIntyre K, Hole DJ, et al. More 'malignant' than cancer? Five-year survival following a first admission for heart failure. Eur J Heart Fail 2001;3:315-22.

5. Roger VL. Epidemiology of heart failure. Circ Res 2013;113:646-59.

6. McMurray JJ, Packer M, Desai AS, et al. Angiotensinneprilysin inhibition versus enalapril in heart failure. $\mathrm{N}$ Engl J Med 2014;371:993-1004.

7. Ponikowski P, Voors AA, Anker SD, et al. 2016 ESC Guidelines for the diagnosis and treatment of acute and chronic heart failure: The Task Force for the diagnosis and treatment of acute and chronic heart failure of the European Society of Cardiology (ESC)Developed with the special contribution of the Heart Failure Association (HFA) of the ESC. Eur Heart J 2016;37:2129-200.

8. Gu J, Noe A, Chandra P, et al. Pharmacokinetics and pharmacodynamics of LCZ696, a novel dual-acting angiotensin receptor-neprilysin inhibitor (ARNi). J Clin Pharmacol 2010;50:401-14.

9. Yancy CW, Jessup M, Bozkurt B, et al. 2017 ACC/ AHA/HFSA Focused Update of the 2013 ACCF/AHA Guideline for the Management of Heart Failure: A Report of the American College of Cardiology/American Heart Association Task Force on Clinical Practice Guidelines and the Heart Failure Society of America. Circulation 2017;136:e137-61.

10. Rodrigues $\mathrm{G}$, Tralhão $\mathrm{A}$, Aguiar $\mathrm{C}$, et al. Is the PARADIGM-HF cohort representative of the real-world heart failure patient population? Rev Port Cardiol (Engl Ed) 2018;37:491-6.

11. Pellicori P, Urbinati A, Shah P, et al. What proportion of patients with chronic heart failure are eligible for sacubitril-valsartan? Eur J Heart Fail 2017;19:768-78.

12. Martens $P$, Beliën $H$, Dupont $M$, et al. Insights into implementation of sacubitril/valsartan into clinical practice. ESC Heart Fail 2018;5:275-83.

13. Packer M, McMurray JJ, Desai AS, et al. Angiotensin receptor neprilysin inhibition compared with enalapril on the risk of clinical progression in surviving patients with heart failure. Circulation 2015;131:54-61.

14. Januzzi JL Jr, Prescott MF, Butler J, et al. Association of Change in N-Terminal Pro-B-Type Natriuretic Peptide Following Initiation of Sacubitril-Valsartan Treatment With Cardiac Structure and Function in Patients With Heart Failure With Reduced Ejection Fraction. JAMA 2019;322:1085-95.

15. Gaziano TA, Fonarow GC, Claggett B, et al. Costeffectiveness Analysis of Sacubitril/Valsartan vs Enalapril in Patients With Heart Failure and Reduced Ejection Fraction. JAMA Cardiol 2016;1:666-72.

16. Ollendorf DA, Sandhu AT, Pearson SD. SacubitrilValsartan for the Treatment of Heart Failure: Effectiveness and Value. JAMA Intern Med 2016;176:249-50.

17. McMurray JJV, Trueman D, Hancock E, et al. Costeffectiveness of sacubitril/valsartan in the treatment of heart failure with reduced ejection fraction. Heart 2018;104:1006-13.

18. Liang L, Bin-Chia Wu D, Aziz MIA, et al. Costeffectiveness of sacubitril/valsartan versus enalapril in patients with heart failure and reduced ejection fraction. J Med Econ 2018;21:174-81.

19. Sandhu AT, Ollendorf DA, Chapman RH, et al. CostEffectiveness of Sacubitril-Valsartan in Patients With Heart Failure With Reduced Ejection Fraction. Ann Intern Med 2016;165:681-9.

20. Ramos IC, Versteegh MM, de Boer RA, et al. Cost Effectiveness of the Angiotensin Receptor Neprilysin Inhibitor Sacubitril/Valsartan for Patients with Chronic Heart Failure and Reduced Ejection Fraction in the Netherlands: A Country Adaptation Analysis Under the Former and Current Dutch Pharmacoeconomic Guidelines. Value Health 2017;20:1260-9.

21. Torrado J, Cain C, Mauro AG, et al. Sacubitril/Valsartan 
Averts Adverse Post-Infarction Ventricular Remodeling and Preserves Systolic Function in Rabbits. J Am Coll Cardiol 2018;72:2342-56.

22. Selvaraj S, Claggett B, Pozzi A, et al. Prognostic Implications of Congestion on Physical Examination Among Contemporary Patients With Heart Failure and Reduced Ejection Fraction: PARADIGM-HF. Circulation 2019;140:1369-79.

23. Damman K, Gori M, Claggett B, et al. Renal Effects and Associated Outcomes During Angiotensin-Neprilysin Inhibition in Heart Failure. JACC Heart Fail 2018;6:489-98.

24. Desai AS, Solomon SD, Shah AM, et al. Effect of Sacubitril-Valsartan vs Enalapril on Aortic Stiffness in Patients With Heart Failure and Reduced Ejection Fraction: A Randomized Clinical Trial. JAMA

Cite this article as: Cabral J, Vasconcelos $\mathrm{H}$, Maia-Araújo P, Moreira E, Campelo M, Amorim S, Sousa A, Moura B, Pinto R, Dias C, Silva-Cardoso J. Sacubitril/valsartan in everyday clinical practice: an observational study based on the experience of a heart failure clinic. Cardiovasc Diagn Ther 2021;11(6):12171227. doi: $10.21037 / \mathrm{cdt}-21-312$
2019;322:1077-84.

25. Damman K, Valente MA, Voors AA, et al. Renal impairment, worsening renal function, and outcome in patients with heart failure: an updated meta-analysis. Eur Heart J 2014;35:455-69.

26. Santos-Gallego CG, Vargas-Delgado AP, RequenaIbanez JA, et al. Randomized Trial of Empagliflozin in Nondiabetic Patients With Heart Failure and Reduced Ejection Fraction. J Am Coll Cardiol 2021;77:243-55.

27. Santos-Gallego CG, Requena-Ibanez JA, San Antonio R, et al. Empagliflozin Ameliorates Diastolic Dysfunction and Left Ventricular Fibrosis/Stiffness in Nondiabetic Heart Failure: A Multimodality Study. JACC Cardiovasc Imaging 2021;14:393-407. 


\section{Supplementary}

\section{Appendix 1}

\section{Methods}

A linear regression model was used to assess the change in left ventricular ejection fraction (LVEF) over time after initiation of sacubitril/valsartan.

\section{Results}

The duration of sacubitril/valsartan treatment was positively associated with the magnitude of LVEF improvement during follow-up. A linear regression model evidenced an absolute 6.6\% LVEF increase per year of sacubitril/valsartan treatment $(\mathrm{P}=0.012)$.

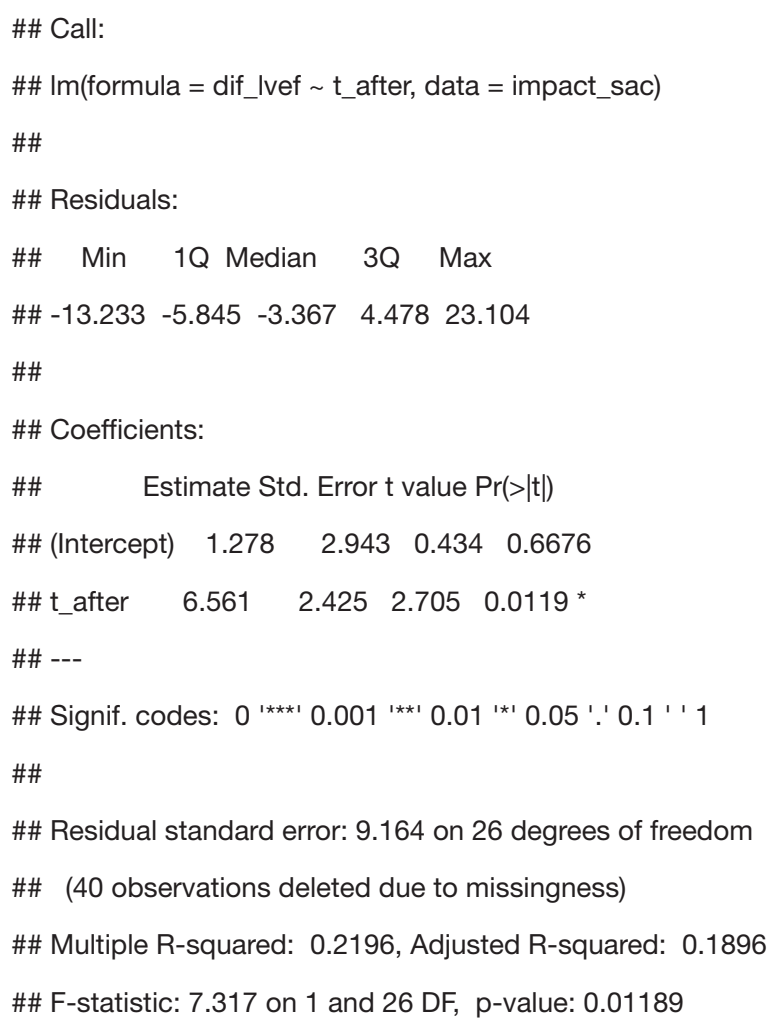

Figure S1 Regression model output, R 3.6.2. dif_lvef, difference of LVEF (\%); t_after, time after initiating treatment (months); LVEF, left ventricular ejection fraction. 